\title{
Evolutionary dynamics of HIV-1 subtype C accessory and regulatory genes in primary infection
}

\author{
R Rossenkhan ${ }^{1 *}$, V Novitsky ${ }^{2}$, TK Sebunya ${ }^{3}$, R Musonda $^{4}$, BA Gashe $^{3}$, M Essex $^{2}$ \\ From AIDS Vaccine 2012 \\ Boston, MA, USA. 9-12 September 2012
}

\section{Background}

Studies addressing the dynamics of accessory and regulatory viral gene diversity and selection during early stage of HIV-1 infection are limited but crucial for progress towards vaccine research.

\section{Methods}

Intra-patient diversity and evolution was assessed during primary HIV-1C infection, viral quasispecies were obtained by single genome amplification (SGA) at multiple sampling time points up to one year post-seroconversion $(\mathrm{p} / \mathrm{s})$.

\section{Results}

The mean intra-patient diversity was found to be $0.11 \%$ (95\%CI; 0.02 to 0.20$)$ for vif, $0.23 \%$ (95\%CI; 0.08 to 0.38 ) for vpr, $0.35 \%$ (95\%CI; -0.05 to 0.75 ) for vpu, $0.18 \%(95 \%$ $\mathrm{CI} ; 0.01$ to 0.35 ) for tat exon 1 and $0.30 \%(95 \% \mathrm{CI} ; 0.02$ to 0.58 ) for rev exon 1 during the time period 0 to 90 days $\mathrm{p} / \mathrm{s}$. The intra-patient diversity increased gradually in all non-structural genes over the first year of HIV-1 infection, which was evident from the vif mean intra-patient diversity of $0.46 \%(95 \% \mathrm{CI} ; 0.28$ to 0.64$)$, vpr $0.44 \%$ (95\%CI; 0.24 to 0.64$)$, vpu $0.84 \%$ (95\%CI; 0.55 to 1.13$)$, tat exon $10.35 \%(95 \% \mathrm{CI} ; 0.14$ to 0.56 ) and $0.42 \%$ (95\%CI; 0.18 to 0.66 ) for rev exon 1 during the time period of 181 to 500 days p/s. Statistically significant increases in viral diversity were observed for vif $(\mathrm{p}=0.013)$ and $\mathrm{vpu}(\mathrm{p}=0.002)$. Weak and sporadic associations between levels of viral diversity within the nonstructural genes and HIV-1 RNA load during primary infection were found. Positive and negative selection patterns over the first year post-seroconversion were assessed in each of these genes, providing insight into the selection pressures on these genes which are crucial for viral replication in-vivo.

\section{Conclusion}

Our study highlights differential diversity and slower diversification across these HIV-1 genes. The most likely cause is different selection pressure imposed by host immune response to the encoded viral gene products that may result in different evolutionary rates.

\section{Author details}

${ }^{1} \mathrm{HSPH} / \mathrm{UB} / \mathrm{BHP}$, Boston, MA, Botswana. ${ }^{2}$ Harvard School of Public Health (HSPH)/ BHP, Boston, MA, USA. ${ }^{3}$ University of Botswana (UB), Gaborone, Botswana. ${ }^{4}$ Botswana Harvard AIDS Institute Partnership (BHP), Gaborone, Botswana.

\section{Published: 13 September 2012}

\section{doi:10.1186/1742-4690-9-S2-P142}

Cite this article as: Rossenkhan et al:: Evolutionary dynamics of HIV-1 subtype $\mathrm{C}$ accessory and regulatory genes in primary infection. Retrovirology 2012 9(Suppl 2):P142. 